\title{
Influencia del entorno familiar y escolar en la práctica de actividad física
}

\author{
Influence of family and school environment on children's physical activity
}

\author{
Natalia Abalde-Amoedo, Margarita R. Pino-Juste \\ Didáctica, Organización Escolar y Métodos de Investigación, Universidad de Vigo, España
}

\begin{abstract}
Resumen
La inactividad física ocupa el cuarto puesto entre los factores de riesgo de mortalidad a nivel mundial. Tras una revisión bibliográfica exhaustiva sobre esta temática a nivel internacional, los resultados más destacables permiten confirmar una correlación positiva entre las familias cuyos miembros se involucran en la actividad física (AF) de sus menores y unos mayores niveles de $\mathrm{AF}$ vigorosa. También se observa una reducción de comportamientos sedentarios en las horas de tiempo libre cuando las familias dan apoyo a los hijos. Los programas de actividad física implementados en el ámbito escolar han demostrado una mejora en los niveles de AF de los niños. Así mismo, la práctica de AF con compañeros de clase y amigos es, también, un factor determinante.

Palabras clave: influencia de la familia, relación familia-escuela, nivel de actividad física
\end{abstract}

\begin{abstract}
Physical inactivity ranks fourth among the mortality risk factors in the World. After carrying out an exhaustive literature review of relevant articles on this subject at international level, the most remarkable results confirm a positive correlation between families whose members are involved in their children's physical activity (PA) and higher levels of vigorous PA. At the same time, a reduction of sedentary behaviours in children's leisure time is observed. Existing school programmes based on physical activity might improve PA levels in the students. Also, practicing PA with their classmates and friends is a determinant factor too.

Keywords: family influence, family school relationship, physical activity level.
\end{abstract}

\section{Introducción}

Según la OMS (2010), la obesidad infantil es uno de los problemas de salud más graves del siglo XXI a nivel mundial. Se estima que más de 44 millones de niños menores de 5 años en todo el mundo superan el umbral del sobrepeso (OMS, 2014).

La inactividad física es uno de los principales desencadenantes de la obesidad infantil, conjuntamente con los hábitos alimenticios, y en la actualidad ocupa el cuarto puesto entre los factores de riesgo de mortalidad a nivel mundial, tan sólo precedido por la hipertensión, la diabetes mellitus y las dislipemias. Además, los comportamientos sedentarios en las personas pueden provocar la aparición temprana de éstas y otras enfermedades no transmisibles (ENT) e incluso patologías de carácter psicológico.

Según la Organización Mundial de la Salud, las ENT provocan, sólo en Europa, el 86\% de las defunciones.
De ahí que se haya situado el foco sobre la actividad física, un método no farmacológico accesible a un elevado porcentaje de la población mundial. Esto permitiría, en primer lugar, mejorar la salud general de la población y, en segundo, reducir los gastos de los sistemas de salud de países desarrollados entre un 1 y un 2,6\% (Pratt, Norris, Lobelo, Roux y Wang, 2014).

Cabe, entonces, preguntarse por qué los niveles de práctica de actividad física son tan bajos en la población general y específicamente en los niños, de cuyos hábitos y estilos de vida son responsables las familias y la educación, puesto que la mayor parte de su tiempo en la etapa infantil la pasan a cargo de miembros de su familia o en la escuela.

Además, la exposición de los niños a los entornos en los que desarrollan su vida cotidiana en edades tempranas influye directamente en su estilo de vida y salud en la etapa adulta (Dundas, Leyland y MacIntyre, 2014). Esto comprendería, como se ha comentado anteriormente, el entorno familiar, escolar e incluso el área de residencia. Se podría pensar, por tanto, que ambos entornos también van a influir en cuestiones específicas de la vida de los niños, como el fomento de la práctica de actividad física.

En España, el sistema educativo no llega a cubrir todos los aspectos necesarios para el fomento de la actividad física en edades tempranas. A pesar de que cada vez se están presentando más programas y guías para consolidar estos valores saludables en los niños, los esfuerzos son todavía insuficientes, y pierden importancia a medida que los estudiantes avanzan hacia la educación superior. Por otra parte, el estilo de vida actual de gran parte de la población junto a los avances científicos y tecnológicos han separado a las familias de los estilos de vida activos de antaño. Las mejoras en los medios de transporte, los cambios producidos en las actividades desarrolladas en los espacios de tiempo libre, los cambios económicos, laborales, etc. han provocado cambios que impiden, o dificultan en gran medida la práctica y el fomento de la actividad física.

Por tanto, la solución pasa por descubrir formas que permitan cambiar los patrones actuales de comportamiento de las nuevas generaciones, tratando de transformar sus conductas por medio de las actuaciones en el contexto familiar y en la colaboración de los sistemas educativos, profesores, compañeros, etc.

\section{Método}

\section{Diseño}

Para este estudio retrospectivo ex post facto (Montero y León, 2002), se realizó una búsqueda de los artículos en 
las siguientes bases de datos internacionales: Web of Science (WoS), Scopus y Google Scholar.

Se analizaron un total de 24 documentos científicos. En primer lugar, se realizó una búsqueda de artículos científicos y revisiones bibliográficas en la base de datos Scopus, tanto en español como en inglés, mediante varias ecuaciones de búsqueda y limitando la búsqueda a documentos publicados a partir del año 2004. De dichos documentos se extrajeron referencias utilizadas en los mismos y potencialmente relevantes en nuestro campo de revisión. El mismo proceso se siguió en la base de datos WoS. Por último se realizó una búsqueda en Google Scholar para localizar algunos artículos concretos y material adicional $\mathrm{y}$ en páginas de instituciones internacionales de reconocido prestigio en el ámbito de estudio, para obtener algunas guías e información complementaria.

\section{Criterios de inclusión-exclusión}

Se incluyeron los artículos que ofrecían resultados relevantes para la temática estudiada. Se excluyeron aquellos que no incluían información sobre las influencias de familia/escuela sobre la AF de los niños, o aquellos que ofrecían resultados confusos.

\section{Resultados}

\section{Relación familia-actividad física}

Recientemente, y ante la creciente importancia que la promoción de la salud por medio de la actividad física está adquiriendo, numerosos investigadores están centrando sus trabajos determinar todos los aspectos que rodean esta temática. El aspecto social cobra gran importancia, y la familia, como principal agente de socialización de los niños, es un aspecto clave.

Diversos estudios han correlacionado la práctica de AF de niños y adolescentes con el apoyo recibido por parte de sus familias, ya sea en forma de estímulos o involucrándose en la práctica (Holm, Wyatt, Murphy, Hill y Ogden, 2012; Pharr, Irwin e Irwin, 2014; Trost, Sallis, Pate, Freedson,Taylor y Dowda, 2003)

Autores como Wang, Liu, Ren, Lv y Li (2015), confirmaron que las familias que se involucran en la actividad física de los niños ejercen una mayor influencia en sus comportamientos que las familias que sólo los apoyan verbalmente u observándolos durante la práctica. A pesar de ello, estos últimos comportamientos también se mostraron eficaces a la hora de reducir los comportamientos sedentarios en las horas de tiempo libre de los niños. El estudio fue llevado a cabo con un total de 7286 estudiantes, de los cuales menos del 10\% participaban en actividades físicas moderadas a vigorosas en ese momento.

En este sentido, Martín-Matillas et al. (2012) profundizaron un poco más en esta cuestión, siendo capaces de encontrar asociaciones entre miembros concretos de la familia y el nivel de participación de los jóvenes en AF. En el estudio participaron un total de 3288 adolescentes, de los que se obtuvieron datos válidos en al menos una de las tres variables de la condición física estudiada: fuerza muscular, velocidad/agilidad y capacidad cardiorrespiratoria. Los casos en los que el padre, madre, hermano o mejor amigo estaban involucrados en la AF correlacionaron positivamente con una mejor capacidad cardiorrespiratoria. Cuando las madres o las hermanas eran las que tenían el compromiso con la práctica de AF se observaron mayores niveles de fuerza muscular. Cuando los padres eran los que apoyaban a sus hijos en la práctica, las tres variables de la condición física analizadas mostraron mejores resultados. Finalmente concluyeron que las intervenciones encaminadas a mejorar la condición física de los niños son más efectivas si se involucran, particularmente, a las madres y padres de los mismos.

A pesar de estos hallazgos, Brockman et al. (2009) aseguran que partiendo de la base que la familia es clave para el estímulo de los niños hacia la práctica de actividad física regular, familias de diferentes contextos socioeconómicos apoyarán a sus niños de diferente forma, por lo que el diseño de intervenciones en AF que incluyan el trabajo con familias deberán realizarse a medida del grupo socio-económico con el que se vaya a trabajar. Los participantes de grupos socioeconómicos medios-altos eran asistidos a través de apoyo financiero y logístico, la coparticipación y el modelado . Los padres de los grupos socioeconómicos más bajos, sin embargo, apoyaban a sus niños de forma verbal o con exigencias.

Tras haber mencionado algunos de los diferentes tipos de apoyo que pueden recibir los niños por parte de sus familias, tiene sentido mencionar la clasificación realizada por Beets, Cardinal y Alderman (2010) tras la realización de una revisión sistemática de artículos de esta temática. Ellos distinguen, en primer lugar, entre dos tipos de mecanismos de apoyo: tangibles e intangibles. El apoyo tangible se divide a su vez en dos categorías: la instrumental, que correspondería a la compra de equipamiento y el pago de las tasas junto al transporte de los niños al punto de práctica de AF y la condicional, que correspondería a la práctica de actividad física con los niños y la supervisión de la misma. Por otra parte, el apoyo intangible se divide en otras dos categorías: motivacional, que correspondería a la aportación de estímulos alabanzas y la informacional.

La gran mayoría de los estudios revisados por estos autores mostraron una correlación positiva entre ambos tipos de apoyo y la práctica de AF por parte de los niños. Sin embargo, diferentes tipos o niveles de apoyo producirán diferentes niveles de motivación en los niños hacia la práctica de AF (Wang, Liu, Ren, Lv y Li, 2015).

Los resultados de otras publicaciones sugieren que algunas acciones de los padres, como el nivel de control o de estímulo para la práctica de AF están directamente relacionadas con los niveles de actividad física moderada a vigorosa y el descenso lineal en la práctica de AF por parte de los niños (Bradley, McRitchie, Houts, Nader y O'Brien, 2011).

Estos y otros autores (Brockman et al., 2009) hallaron cambios en el nivel de AF de los niños según se acercan a la pubertad, encontrando diferencias significativas según el enfoque que los padres lleven a cabo.

Un estudio reciente llevado a cabo por Maitland, Stratton, Foster, Braham y Rosenberg (2014), destacó la importancia que el espacio físico de los hogares tiene sobre las oportunidades de práctica de AF. Indicaron que 
factores como el espacio total de la vivienda, el diseño, la localización, el equipamiento, la seguridad percibida en la casa y otros factores tienen influencia sobre el nivel de actividad física. Comentan, por ejemplo, que en casas en las que el espacio del jardín es pequeño las oportunidades de práctica de AF se reducen, mientras que en casas que albergan múltiples salones o salas, los aparatos multimedia se multiplican, produciendo el mismo resultado.

A pesar de que se ha demostrado que los factores relativos a la familia y el hogar y la práctica de actividad física de los niños están asociados, autores como McMinn, Griffin, Jones y van Sluijs (2012) han investigado la posible dependencia de los resultados al tiempo de práctica, tratando de observar las diferencias para los períodos tras la jornada escolar durante la semana y los períodos de fines de semana. Los resultados probaron que el apoyo familiar está asociado con la práctica de AF fuera de la escuela a lo largo de toda la semana, incluidos los fines de semana.

Por tanto, y según los resultados revisados hasta el momento, la familia es un factor clave en la práctica de actividad física por parte de los niños. Y aunque diferentes niveles/tipos de apoyo producirán diferentes respuestas en los niños es evidente que el apoyo o participación de las mismas estimulará siempre la práctica de AF. En el caso de realizar intervenciones que involucren a la familia, será imprescindible analizar el contexto y adaptarse a él para lograr la mayor eficacia posible.

\section{Relación escuela-actividad física}

La escuela se erige como el otro gran agente de socialización de las personas durante su etapa infantil. En la mayoría de los casos, el único tiempo que pasan fuera del entorno familiar lo hacen en la escuela. Allí se integrarán en la comunidad y adquirirán una educación social.

Edwards et al. (2015) encontraron, en un estudio llevado a cabo con 56 padres de niños con edades comprendidas entre 5 y 6 años, que los primeros tienen la firme creencia de que los amigos de sus hijos generan una importante influencia en el tipo de AF en el que sus hijos quieren participar. Esto confirmaría los resultados obtenidos en otro estudio (Jago et al., 2011), en el que los autores encontraron que los niños que tienen amigos y activos y que las niñas que participan en actividades físicas con sus mejores amigas presentan mayores niveles de práctica de actividad física moderada a vigorosa. Además, si los niños participan en actividades físicas en su casa o vecindario junto a sus mejores amigos, presentarán mayores niveles de AF.

Ya son numerosos los estudios que asocian la participación en las clases de educación física, la práctica de actividad física y el desarrollo de programas de actividad física en la escuela con unos niveles más altos de AF en la vida cotidiana de los estudiantes (Duncan, Strycker y Chaumeton, 2015; Racette et al., 2015).

Los programas llevados a cabo en la escuela basados en la actividad física permiten mejorar los niveles de AF de los estudiantes (Kahlin, Werner y Alricsson, 2014). Así mismo, Arto (2015) observó que mediante programas de actividad física se puede evitar el descenso de los niveles de AF, aunque afirma que se debe prestar atención a las horas de tiempo libre fuera de la escuela de los niños, principalmente tras las horas lectivas y los fines de semana.

Además, las escuelas que ofrecen un mayor número de actividades deportivas se erigen como estrategias efectivas para ayudar a los adolescentes a alcanzar las recomendaciones mínimas sobre actividad física (Fuller, Sabiston, Karp, Barnett y O'Loughlin, 2011).

Por último, Panter, Jones, Van Slujis y Griffin (2011) afirmaron que los estudiantes que realizan los trayectos entre su hogar y la escuela caminando o en bicicleta, presentan mayor disposición a realizar más minutos de actividad física moderada a vigorosa en los días de semana laborables, aunque destacan la importancia de la distancia del trayecto, afirmando que a mayores distancias mayores niveles de AF generales. Este estudio confirmó los resultados obtenidos por uno anterior (Loucaides y Jago, 2008).

\section{Discusión}

Existen evidencias suficientes que permiten confirmar la influencia que ejercen las familias y el entorno escolar sobre la práctica de actividad física de los niños (Bauer, Berge y Neumark-Sztainer, 2011; Harrison, M., Burns, McGuinness, Heslin y Murphy, 2006).

Algunos de los estudios revisados permitían incluso diferenciar entre los diferentes tipos de apoyo que puede dar la familia, que variarán en base al contexto socio-cultural y económico de cada familia. Esto indicaría la necesidad de personalizar las intervenciones que involucren a la familia según las necesidades de cada colectivo.

En cuanto a la escuela, se sabe que la influencia sobre la práctica de AF puede ser ejercida dentro del grupo de iguales o por el colectivo docente y el propio sistema educativo.

Aún con las diferencias intra/inter-comunidad y los métodos por los cuales cada individuo reciba dicho apoyo, está confirmado que ambos entornos son potentes herramientas para un correcto desarrollo de los niños en el ámbito de la actividad física.

Por tanto, la necesidad principal se centra ahora en conocer qué parte del proceso está fallando, ya que los índices de práctica de actividad física parecen ser cada vez más bajos a pesar de conocer métodos eficaces para estimular la práctica de AF. Algunos autores confirman que la proporción de niños, de edades comprendidas entre 13 y 15 años, que no realizan actividad física moderada a vigorosa durante, al menos, 60 minutos al día se encuentra entorno al 80\% (Hallal et al., 2012).

Por lo tanto, parece importante el diseño y la evaluación de programas de actividad física con una intensidad moderada e intensa en el entorno comunitario y escolar vinculando siempre el apoyo y la colaboración de las familias en estos programas.

\section{Referencias}

Arto, G. (2015). Children's segment specific light physical activity across two years of school-based 
program. Journal of Physical Education and Sport, 15(1), 88. doi: 10.7752/jpes.2015.01015

Bauer, K. W., Berge, J. M., \& Neumark-Sztainer, D. (2011). The importance of families to adolescents' physical activity and dietary intake. Adolescent medicine: state of the art reviews, 22(3), 601-13.

Beets, M. W., Cardinal, B. J., \& Alderman, B. L. (2010). Parental social support and the physical activityrelated behaviors of youth: a review. Health Education \& Behavior, 37 (5), pp. 621-644. doi: 10.1177/1090198110363884

Bradley, R. H., McRitchie, S., Houts, R. M., Nader, P., \& O'Brien, M. (2011). Parenting and the decline of physical activity from age 9 to 15 . International Journal of Behavioral Nutrition and Physical Activity, 8(1), 33. doi: 10.1186/1479-5868-8-33

Brockman, R., Jago, R., Fox, K. R., Thompson, J. L., Cartwright, K., \& Page, A. S. (2009). Get off the sofa and go and play": family and socioeconomic influences on the physical activity of 10-11 year old children. BMC public health, 9(253), 1-7. doi: 10.1186/1471-2458-9-253

Duncan, S. C., Strycker, L. A., \& Chaumeton, N. R. (2015). School influences on the physical activity of african american, latino, and white girls. Journal of school health, 85(1), 43-52. doi: 10.1111/josh.12218

Dundas, R., Leyland, A.H. \& MacIntyre, S. (2014). Early-life school, neighborhood, and family influences on adult health: A multilevel cross-classified analysis of the aberdeen children of the 1950s study. American Journal

Epidemiology, 180 (2), pp. 197-207. doi: 10.1093/aje/ kwu110

Edwards, M. J., Jago, R., Sebire, S. J., Kesten, J. M., Pool, L., \& Thompson, J. L. (2015). The influence of friends and siblings on the physical activity and screen viewing behaviours of children aged 5-6 years: a qualitative analysis of parent interviews. BMJ Open, 5 (5), art. no. e006593. doi: 10.1136/bmjopen-2014-006593

Fuller, D., Sabiston, C., Karp, I., Barnett, T., \& O'Loughlin, J. (2011). School sports opportunities influence physical activity in secondary school and beyond. Journal of School Health, 81(8), 449-454. doi: 10.1111/j.1746-1561.2011.00613.x

Hallal, P. C., Andersen, L. B., Bull, F. C., Guthold, R., Haskell, W., Ekelund, U., \& Lancet Physical Activity Series Working Group. (2012). Global physical activity levels: surveillance progress, pitfalls, and prospects. The lancet, 380(9838), 247-257. doi: http://dx.doi.org/10.1016/S0140-6736(12)60646-1

Harrison, M., Burns, C. F., McGuinness, M., Heslin, J., \& Murphy, N. M. (2006). Influence of a health education intervention on physical activity and screen time in primary school children:'Switch Off-Get Active'. Journal of Science and Medicine in Sport, 9(5), 388-394. doi: 10.1016/j.jsams.2006.06.012

Holm, K. E., Wyatt, H. R., Murphy, J. R., Hill, J. O., \& Ogden, L. G. (2012). Parental influence on child change in physical activity during a family-based intervention for child weight gain prevention. Journal of physical activity \& health, 9(5), 661.
Jago, R., Macdonald-Wallis, K., Thompson, J. L., Page, A. S., Brockman, R., \& Fox, K. R. (2011). Better with a buddy: influence of best friends on children's physical activity. Medicine and Science in Sports and Exercise, 43(2), pp. 259-265. doi: 10.1249/MSS.0b01 3e3181edefaa

Kahlin, Y., Werner, S., \& Alricsson, M. (2014). A physical activity program for Swedish physically inactive female high school students: a controlled intervention study. Journal of physical activity \& health, 11(6), 1162-1169. doi: 10.1123/jpah.2012-0321

Loucaides, C.A., \& Jago, R. (2008). Differences in physical activity by gender, weight status and travel mode to school in Cypriot children. Preventive Medicine 47(1), 107-111

Maitland, C., Stratton, G., Foster, S., Braham, R., \& Rosenberg, M. (2014). The Dynamic Family Home: a qualitative exploration of physical environmental influences on children's sedentary behaviour and physical activity within the home space. International Journal of Behavioral Nutrition and Physical Activity, 11(1), 157. doi: 10.1186/s12966-014-0157-1

Martín-Matillas, M., Ortega, F. B., Ruiz, J. R., Martínez-Gómez, D., Vicente-Rodríguez, G., Marcos, A., ... \& Castillo on behalf of the HELENA Study, M. J. (2012). Active relatives and health-related physical fitness in European adolescents: The HELENA Study. Journal of sports sciences, 30(13), 1329-1335. doi: 10.1080/02640414.2012.710758

McMinn, A. M., Griffin, S. J., Jones, A. P., \& van Sluijs, E. M. (2012). Family and home influences on children's after-school and weekend physical activity. The European Journal of Public Health, 23 (5), pp. 805-810. doi: 10.1093/eurpub/cks160

Montero, I. y León, O. G. (2002). Clasificación y descripción de las metodologías de investigación en Psicología. International Journal of Clinical and Health Psychology, 2, 503-508.

Organización Mundial de la Salud (2010). Recomendaciones mundiales sobre actividad física para la salud. Ginebra: Organización Mundial de la Salud.

Organización Mundial de la Salud (2014). Estadísticas sanitarias mundiales 2014. Ginebra: Organización Mundial de la Salud.

Pharr, J., Irwin, C. C., \& Irwin, R. L. (2014). Parental Factors That Influence Swimming in Children and Adolescents. International Journal of Aquatic Research and Education, 8(4), 368. doi: 10.1123/ijare.2014-0041

Pratt, M., Norris, J., Lobelo, F., Roux, L. y Wang, G. (2014). The cost of physical inactivity: moving into the 21st century. British journal of sports medicine, 48(3), 171-173. doi: 10.1136/bjsports-2012-091810

Racette, S. B., Dill, T. C., White, M. L., Castillo, J. C., Uhrich, M. L., Inman, C. L., ... \& Clark, B. R. (2015). Peer Reviewed: Influence of Physical Education on Moderate-to-Vigorous Physical Activity of Urban Public School Children in St. Louis, Missouri, 20112014. Preventing chronic disease, 12 (3), art. no. 140458 . doi: $10.5888 /$ pcd12.140458 
Trost, S. G., Sallis, J. F., Pate, R. R., Freedson, P. S., Taylor, W. C., \& Dowda, M. (2003). Evaluating a model of parental influence on youth physical activity. American journal of preventive medicine, 25(4), 277-282.

Wang, X., Liu, Q. M., Ren, Y. J., Lv, J., \& Li, L. M. (2015). Family influences on physical activity and sedentary behaviours in Chinese junior high school students: a cross-sectional study. BMC public health, 15(1), 287. doi: 10.1186/s12889-015-1593-9

Whitaker, R. C., Wright, J. A., Pepe, M. S., Seidel, K. D. \& Dietz, W. H. (1997). Predicting obesity in young adulthood from childhood and parental obesity. New England Journal of Medicine, 337(13), 869-873. 